from treatment exposure. Treatments were categorized into TNF and non-TNF, which included conventional DMARDs and other non-TNF biologics. Multivariate linear regression analyses were used to evaluate impact of BMI on treatment outcomes controlling for baseline covariates of age, sex, disease duration, comorbidities, baseline disease activity and serostatus. Separate models were run for the TNF and non-TNF groups.

Results: A total of $997(78 \%)$ pts in the registry had baseline BMI values and were included in the analysis. Around $37 \%(n=371)$ had TNF exposure and were included in the TNF cohort; the remainder $(63 \%$; $n=626)$ were included in the non-TNF cohort. Proportions of pts in the normal, overweight and obese groups for the TNF cohort were $45.5 \%(n=169), 27.5 \% \quad(n=102)$ and $27.0 \%(n=100)$, respectively. For the non-TNF cohort, these were $41.7 \%(n=261), 33.1 \%(n=207)$ and $25.2 \%(n=158)$, respectively. In both cohorts, pts with normal BMls were younger vs the overweight and obese BMI groups. However, obese BMI pts had higher disease activity measures at baseline (mean [SD] CDAl: 22.8 [17.8] for TNF and 24.9 [17.3] for non-TNF) vs the normal BMI pts (17.5 [15.9] for TNF and 19.9 [16.7] for non-TNF) and overweight BMI pts (20.9 [16.5] for TNF and 20.5 [15.0] for non-TNF). Adjusted mean change from baseline in disease activity in the TNF cohort was significantly reduced across all disease activity measures for the normal BMI group $(p<0.05)$, but not for the overweight and obese groups (Fig). There were significant reductions in disease activity measures for all BMl groups (all $p<0.05$ ) in the non-TNF cohort (Fig).

Figure. Association between BMI and Treatment Outcomes DAS28 (CRP), CDAI, SDAI and Joint Count
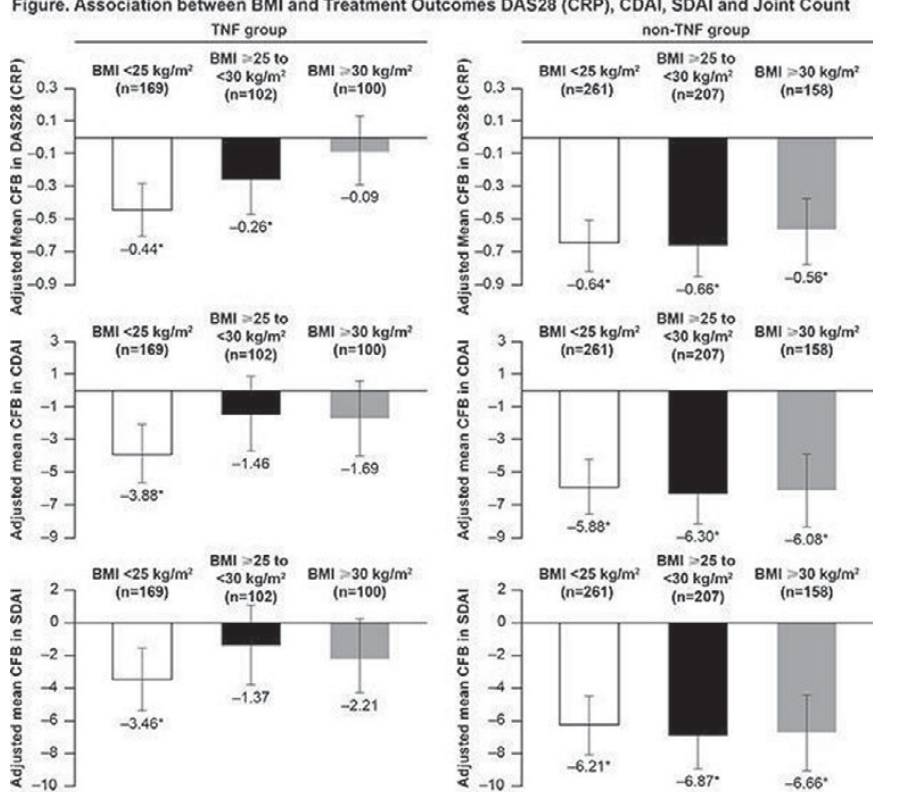

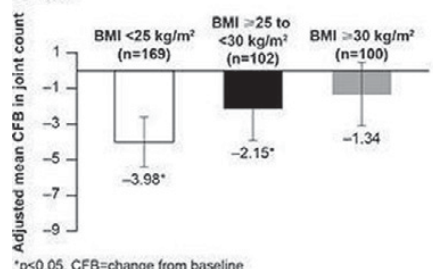

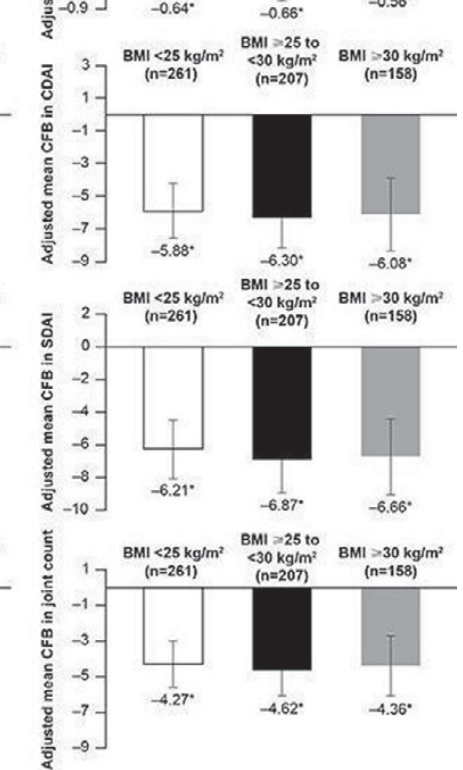

Conclusions: Independent of BMI, non-anti-TNF therapy demonstrated similar outcomes in pts with RA. However, obese and overweight pts with RA (vs normal weight) had less improvement in disease activity (as measured by DAS28 [CRP]) with anti-TNF therapy.

References:

[1] Gremese E, et al. Arthritis Care Res 2013:65:94-100.

[2] Klaasen R, et al. Arthritis Rheum 2011;63:359-64.

[3] Gardette A, et al. Ann Rheum Dis 2015;74:1041.

Disclosure of Interest: E. Alemao Shareholder of: Bristol-Myers Squibb, Employee of: Bristol-Myers Squibb, Z. Guo Shareholder of: Bristol-Myers Squibb, Employee of: Bristol-Myers Squibb, C. lannaccone: None declared, M. Frits: None declared, M. Weinblatt Grant/research support from: Amgen, Bristol-Myers Squibb, Crescendo Biosciences, UCB, DxTerity, Consultant for: Amgen, BristolMyers Squibb, Crescendo Biosciences, UCB, AbbVie, Lilly, Pfizer, Roche, N. Shadick Grant/research support from: Bristol-Myers Squibb, UCB, Mallinckrodt, Amgen, Brescendo Biosciences, Consultant for: Bristol-Myers Squibb DOI: 10.1136/annrheumdis-2017-eular.1566

\section{SAT0198 TOCILIZUMAB FOR THE MANAGEMENT OF RHEUMATOID ARTHRITIS: DISCONTINUATION DUE TO INEFFICACY AND TOXICITY - EXPERIENCE FROM A LARGE TEACHING HOSPITAL}

E. Byrne, P. Mark, S. Khalid, K.-P. Kuet, R. Kilding, K. Graves, J. Maxwell, M. Akil. Rheumatology, Sheffield Teaching Hospitals, Sheffield, United Kingdom

Background: Tocilizumab (TCZ) is a humanised anti interleukin-6 receptor antibody licensed for use for the treatment of moderate to severe Rheumatoid Arthritis (RA) as monotherapy or in combination with methotrexate (MTX).

Objectives: To describe the use of TCZ for RA in a large UK teaching centre and examine reasons for treatment discontinuation.

Methods: A retrospective case note review of all adult patients receiving TCZ either alone or in combination with DMARDS, for the treatment of RA between April 2009 and January 2017 in Sheffield, UK.

Results: 132 patients received TCZ for RA. $71 \%$ were female. $61 \%$ were CCP positive. Mean disease duration was 15.6 years (range 1.5-43). 46 (34.6\%) received TCZ as monotherapy, $55(42.1 \%)$ in combination with MTX and 31 (23.3\%) other DMARDS. $23 \%$ of patients received concomitant oral prednisolone. Median duration of TCZ treatment was 27 months across the whole cohort, and 19 months in those who discontinued treatment.

Overall $44(33 \%)$ patients discontinued TCZ; 5 due to primary and 10 secondary inefficacy, 27 patients due to adverse events ( 8 recurrent infection, 5 abnormal LFT, 4 malignancy, 3 rash, 7 other including 1 death whilst on treatment). A logistic regression model, including gender, smoking status, disease duration, DMARD use, steroid treatment and number of prior biologics was constructed to examine association with treatment discontinuation. Of these factors, disease duration $(p=0.05)$ and number of previous biologics $(p=0.09)$ were weakly associated with persistence of TCZ and in particular there was no association of concomitant DMARD or steroid treatment with discontinuation either due to lack of efficacy or adverse events. Table 1 demonstrates the proportion of patients stopping treatment, and treatment duration according to previous biologic treatment received. We have not seen any cases of infusion reaction, diverticular perforation or reactivation of tuberculosis.

Table 1. Proportion of Patients Continuing TCZ and Treatment Duration According to Previous Biologic Treatment

\begin{tabular}{lccccc}
\hline & & \multicolumn{3}{c}{ Previous Biologics Received } \\
\cline { 3 - 6 } & & 0 & 1 & 2 & 3 or more \\
\hline Continued & N (\%) & $15(83 \%)$ & $31(66 \%)$ & $24(51 \%)$ & $12(60 \%)$ \\
Discontinued & Treatment Duration (Mths) & 46 & 27 & 26 & 58 \\
& N (\%) & $3(17 \%)$ & $16(34 \%)$ & $23(49 \%)$ & $8(40 \%)$ \\
& Treatment Duration (Mths) & 4.5 & 14 & 24 & 18 \\
& Lack of Efficacy/Toxicity & $2 / 1$ & $7 / 9$ & $9 / 14$ & $3 / 5$ \\
\hline
\end{tabular}

${ }^{*}$ To date.

Conclusions: Our real world data on the use of TCZ in the treatment of adult patients with RA is consistent with clinical trial data for efficacy and safety and is similar to other biological drugs used in the treatment of RA. We have seen a relatively low rate of withdrawal due to primary and secondary treatment failure.

Disclosure of Interest: None declared

DOI: 10.1136/annrheumdis-2017-eular.5105

\section{SAT0199 SUBCUTANEOUS TOCILIZUMAB MONOTHERAPY OR COMBINED WITH A CSDMARD IN PATIENTS WITH RHEUMATOID ARTHRITIS: TOZURA, A POOLED ANALYSIS OF PHASE IV STUDIES IN 22 COUNTRIES}

E. Choy ${ }^{1}$, R. Caporali ${ }^{2}$, R. Xavier ${ }^{3}$, B. Fautrel ${ }^{4}$, R. Sanmarti ${ }^{5}$, C. Bernasconi ${ }^{6}$, A. Pethö-Schramm ${ }^{6}$. ${ }^{1}$ Cardiff University, Cardiff, United Kingdom; ${ }^{2}$ University of Pavia, Pavia, Italy; ${ }^{3}$ Universidade Federal do Rio Grande do Sul Porto Alegre, Rio Grande do Sul, Brazil; ${ }^{4}$ Pierre and Marie Curie University, Paris, France; ${ }^{5}$ Universitat de Barcelona, Barcelona, Spain; ${ }^{6}$ F. Hoffmann-La Roche AG, Basel, Switzerland

Background: Tocilizumab administered subcutaneously (TCZ-SC) has been approved for the treatment of rheumatoid arthritis (RA) both as mono- and combination therapy.

Objectives: To evaluate the efficacy and safety of TCZ-SC $162 \mathrm{mg}$ once weekly (qw) as monotherapy or in combination with conventional synthetic DMARDs (csDMARDs) over 24 weeks in adult patients (pts) with moderate to severe RA.

Methods: TOZURA is a multinational, open-label, single-arm umbrella program comprising 7 single-country and 4 regional multicountry protocols (total 22 countries). Pts enrolled were inadequate responders to DMARD, and previous biologic DMARDs were allowed in 8 of 11 protocols. Pts received TCZ-SC 162 mg qw for 24 weeks administered at the investigator's discretion as monotherapy or in combination with a csDMARD. Stable oral NSAIDs and corticosteroids (CS), $\leq 10 \mathrm{mg} /$ day prednisone or equivalent, were allowed. Efficacy and safety were evaluated at weeks 1, 2, 4 and every 4 weeks for 24 weeks (plus 8 weeks for safety). Propensity score-based matching was used for between-group tests.

Results: Of 1804 pts treated, $353(19.6 \%)$ received monotherapy (mono) and $1451(80.4 \%)$ combination therapy (combo); 349 pts (19.3\%) had received a prior biologic DMARD. Background characteristics: $81.6 \%$ female; mean age, 54.1 